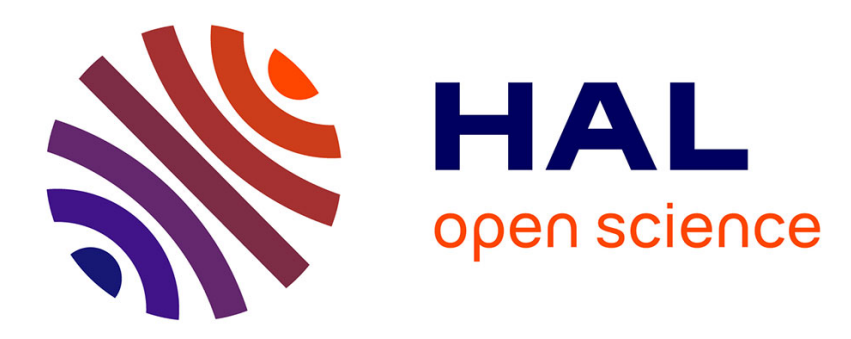

\title{
Stationary convection-diffusion between two co-axial cylinders
}

\author{
Franck Plouraboué, Charles Pierre
}

\section{To cite this version:}

Franck Plouraboué, Charles Pierre. Stationary convection-diffusion between two co-axial cylinders. International Journal of Heat and Mass Transfer, 2007, vol. 50, pp. 4901-4907. 10.1016/j.ijheatmasstransfer.2007.06.037 . hal-00959367

\section{HAL Id: hal-00959367 https://hal.science/hal-00959367}

Submitted on 14 Mar 2014

HAL is a multi-disciplinary open access archive for the deposit and dissemination of scientific research documents, whether they are published or not. The documents may come from teaching and research institutions in France or abroad, or from public or private research centers.
L'archive ouverte pluridisciplinaire HAL, est destinée au dépôt et à la diffusion de documents scientifiques de niveau recherche, publiés ou non, émanant des établissements d'enseignement et de recherche français ou étrangers, des laboratoires publics ou privés. 


\title{
Stationary convection-diffusion between two co-axial cylinders
}

\author{
F. Plouraboué ${ }^{\mathrm{a}, *}$, C. Pierre ${ }^{\mathrm{b}}$ \\ ${ }^{a}$ Institut de Mécanique des Fluides de Toulouse, UMR CNRS-INPT/UPS No. 5502, Avenue du Professeur Camille Soula, 31400 Toulouse, France \\ ${ }^{\mathrm{b}}$ Laboratoire de mathématiques appliquées de Pau, Université de Pau et des Pays de l'Adour, avenue de l'Université, BP 1155, 64013 PAU Cedex, France
}

\begin{abstract}
In this note, we examine the high Péclet number limit of the stationary extended Graetz problem for which two families of real and imaginary eigenvalues are associated, respectively, with a downstream convective relaxation and the upstream diffusive establishment. The asymptotic behavior of both families of eigenvalues is studied, in the limit of large Péclet number and thin wall, which bring to the fore a single parameter dependence, previously mentioned in the literature from numerical investigations [M.A. Cotton, J.D. Jackson, in: R.W. Lewis, K. Morgan (Eds.), Numerical Methods in Thermal Problems, vol. IV, Pineridge Press, Swansea, 1985, pp. 504-515]. The fully developed region is specifically studied thanks to the first eigenvalue dependence on the Péclet number, on the thermal conductivity coefficients and on the diameter ratio of the cylinders. The effective transport between the fluid and the solid is investigated through the evaluation of the fully developed Nusselt number and experimental measurements.
\end{abstract}

Keywords: Micro-systems; Heat transfer; Spectral analysis; Asymptotic

\section{Introduction}

We hereby analyze some special limit of the extended Graetz problem using variable separation eigenfunctions. The mathematical and numerical solutions for this problem has been obtained in numerous previous contribution $[5,6,11,10,9]$. From the precursory contributions of Papoutsakis et al. [5,6], a complete representation of the solution relies on an orthogonal eigenfunction expansion of this problem, independently from the applied boundary conditions for the external cylinder, the input and the output conditions. The core of any explicit numerical computation of the temperature field relies on the evaluation of a subset of the infinite discrete spectrum of eigenvalues, and eigenfunctions.

In this note we focus on some simple expression for the asymptotic behavior of the solution, which put forward a simple parameter already heuristically proposed in previ-

\footnotetext{
* Corresponding author. Tel.: +335612858 80; fax: +33561285878. E-mail address: plourab@imft.fr (F. Plouraboué).
}

ous contribution. The effective transfer between the tube and the co-axial solid cylinder in the fully developed region is studied through the computation of the Nusselt number in Section 4. A comparison with the available experimental results is discussed in the last section.

\section{General solution and eigenvalue problem}

The extended Graetz problem is considered for two complementary configurations sketched in Fig. 1 that we will subsequently refer to as $a$ and $b$. The following nondimensional variables are introduced to describe the problem:

$\eta=\frac{r}{r_{a}}, \quad \zeta=\frac{z}{r_{a}}, \quad R=\frac{r_{b}}{r_{a}}, \quad P e=\frac{2 U r_{a}}{D^{\Gamma}}$,

where $r_{b}$ is the radius of the solid co-axial cylinder. A fully developed hydrodynamic flow inside the tube is considered. The velocity longitudinal component $u$ along the $z$-axis of the tube has a Poiseuille parabolic profile which is proportional to the mean applied pressure gradient $\partial_{z} P$ 


\section{Nomenclature}

$D^{\mathrm{I}}, D^{\mathrm{II}}$ thermal diffusivity in the fluid (I) and in the solid (II)

$F_{R} \quad$ eigenfunction in the solid cylinder

$G \quad$ Graetz eigenfunction

$I \quad$ dimensionless asymptotic parameter

$J_{0}, J_{1} \quad$ zeroth and first Bessel functions of the first kind

$k^{\mathrm{I}}, k^{\mathrm{II}} \quad$ thermal conductivity coefficients

$Y_{0}, Y_{1}$ zeroth and first Bessel functions of the second kind

$\mathrm{Nu} \quad$ Nusselt number

$S \quad$ surface of the cylinder

$r_{a}, r_{b} \quad$ internal radius of the inner and outer cylinder

$P e \quad$ Péclet number

$r \quad$ radial coordinate

$R=\frac{r_{b}}{r_{a}} \quad$ aspect ratio between the inner and the outer cylinder $u=\frac{1}{4} \partial_{z} P\left(r_{a}^{2}-r^{2}\right)=\frac{2 U}{r_{a}^{2}}\left(r_{a}^{2}-r^{2}\right)$,

$U=\frac{1}{S} \int_{S} u \mathrm{~d} S=\frac{2}{r_{a}^{2}} \int_{0}^{r_{a}} u r \mathrm{~d} r$

where $U$ is the averaged fluid velocity inside the tube having section $S$ and radius $r_{a}$. Only axi-symmetric boundary conditions will be considered in the following. The nondimensional Stationary convection-diffusion of heat is described by

$P e \partial_{\zeta} \Theta=\frac{1}{\eta\left(1-\eta^{2}\right)} \partial_{\eta}\left(\eta \partial_{\eta} \Theta\right)$ in $\mathrm{I}$,

$\Delta \Theta=0$ in II,

where $\Theta=T-T_{0}$ is a relative temperature built on the reference temperature $T_{0}$ in the fluid tube I and the solid region II at $z \rightarrow+\infty$ for configuration 1 a and at $z \rightarrow-\infty$ for configuration $1 b$, as already used in $[5,6]$. We restrict our attention to the solution region where there is an adiabatic isolated solid cylinder, i.e. $\zeta>0$ in configuration $1 \mathrm{a}$, and $\zeta<0$ in configuration $1 \mathrm{~b}$

$\partial_{\eta} \Theta^{\mathrm{II}}(R, \zeta)=0$.

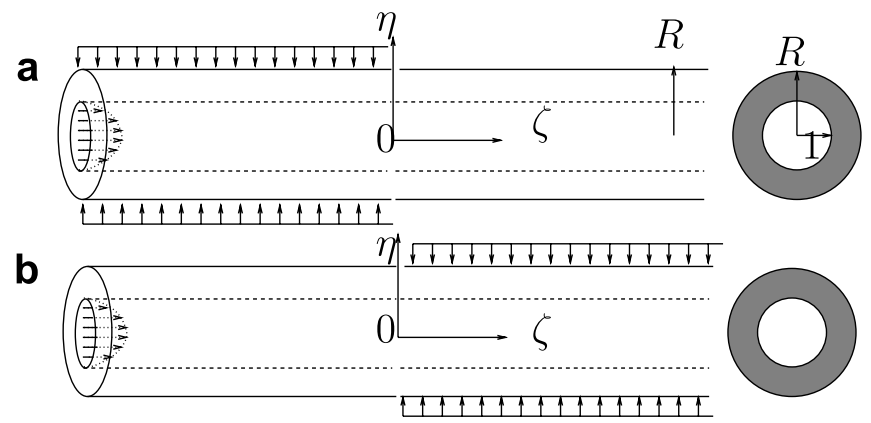

Fig. 1. Schematic representation of the two symmetrical configurations under study.
$P \quad$ fluid pressure

$S \quad$ surface of the inner pipe

$T \quad$ temperature

$T_{0} \quad$ reference temperature at infinity

$\Theta=T-T_{0}$ intrinsic temperature

$U \quad$ averaged longitudinal velocity

$u \quad$ fluid longitudinal velocity

$z \quad$ longitudinal coordinate

Greek symbols

$\eta=\frac{r}{r_{a}} \quad$ dimensionless radial coordinate

$\lambda \quad r_{a} \quad$ eigenvalue of the coupled thermic problem

$\Phi(a, b, z)$ confluent hyper-geometric function

$\zeta=\frac{z}{r_{a}} \quad$ dimensionless longitudinal coordinate
Firstly we are looking at far field decreasing boundary conditions,

$\Theta^{\mathrm{I}} \rightarrow \Theta^{\mathrm{II}} \rightarrow 0 \quad|\zeta| \rightarrow \infty$

While the temperature and flux equilibrium between the fluid and solid region reads

$k^{\mathrm{I}} \partial_{\eta} \Theta^{\mathrm{I}}(1, \zeta)=k^{\mathrm{II}} \partial_{\eta} \Theta^{\mathrm{II}}(1, \zeta)$,

$\Theta^{\mathrm{I}}(1, \zeta)=\Theta^{\mathrm{II}}(1, \zeta)$

where $k^{\mathrm{I}}, k^{\mathrm{II}}$ are the thermal conductivity in the fluid and in the solid.

One can find a general solution to the problem (1) by writing it as

$$
\begin{aligned}
& \Theta^{\mathrm{I}}(\eta, \zeta)=\sum_{n} \theta_{n \pm} G\left(\lambda_{ \pm n}, \eta\right) \mathrm{e}^{-\lambda_{ \pm n}^{2} \zeta / P e}, \\
& \Theta^{\mathrm{II}}(\eta, \zeta)=\sum_{n} \theta_{n \pm} F_{R}\left(\frac{\lambda_{ \pm n}^{2}}{P e} \eta\right) \mathrm{e}^{-\lambda_{ \pm n}^{2} \zeta / P e},
\end{aligned}
$$

where

$$
F_{R}\left(\frac{\lambda_{n}^{2}}{P e} \eta\right)=J_{0}\left(\frac{\lambda_{n}^{2}}{P e} \eta\right) Y_{1}\left(\lambda_{n}^{2} \frac{R}{P e}\right)-J_{1}\left(\lambda_{n}^{2} \frac{R}{P e}\right) Y_{0}\left(\frac{\lambda_{n}^{2}}{P e} \eta\right)
$$

is the linear combinations of the Bessel harmonic eigenfunctions [1] which fulfills the radial adiabatic boundary condition (2).

$G(\lambda, \eta)$ is the Graetz function:

$G(\lambda, \eta)=\mathrm{e}^{-\lambda \eta^{2} / 2} \Phi\left(\frac{1}{2}-\frac{\lambda}{4}, 1, \lambda \eta^{2}\right)$,

where $\Phi(a, b, z)$ is the confluent hyper-geometric function [1] (sometimes referred to as ${ }_{1} F_{1}$ ) which possesses two $a$ and $b$ parameters and one variable $z$. Unlike the solution of the Graetz problem, the solutions families (6) explicitly depend on the Péclet number, on the radius ratio of the fluid and solid region and on the conductivity ratio $k^{\mathrm{I}} / k^{\mathrm{II}}$ through condition (4). 
One of the most interesting features of these solutions is that in the region where no flux is applied, one finds a longitudinal exponential decrease in temperature whose characteristic length is directly related to the first eigenvalue of the problem. The forward eigenvalue $\lambda_{+n}$ is associated with the temperature relaxation convected downstream in 1a configuration while the "backward" eigenvalue $\lambda_{-n}$ is associated with the upstream temperature rise induced by the solid conduction in Fig. 1b configuration.

Using the solution family (6), imposing the interface coupling conditions (4) and (5) leads to the following eigenvalue problem on $\lambda_{n}$ :

$\frac{k^{\mathrm{I}} P e}{k^{\mathrm{II}}} \frac{F_{R}\left(\lambda_{n}^{2} / P e\right)}{F_{R}^{\prime}\left(\lambda_{n}^{2} / P e\right)}=\lambda_{n} \frac{\Phi\left(\frac{1}{2}-\frac{\lambda_{n}}{4}, 1, \lambda_{n}\right)}{2 \Phi^{\prime}\left(\frac{1}{2}-\frac{\lambda_{n}}{4}, 1, \lambda_{n}\right)-\Phi\left(\frac{1}{2}-\frac{\lambda_{n}}{4}, 1, \lambda_{n}\right)}$,

where' denotes the derivative of functions with respect to their variables.

\section{Large Péclet number and small diameter limit}

In the following sections the fully developed region where the first eigenfunction is dominant will be our main focus. The region for which the first eigenfunction prevails over all the other ones is given by the $\zeta$ values for which $\left(\lambda_{2}^{2}-\lambda_{1}^{2}\right) \zeta / P e>3$. The amplitude of this fully developed mode is the projection of the concentration field at $\zeta=0$ with the first eigenfunction. We will not be interested by this amplitude in the following, focusing on the first eigenvalue and Nusselt number. We investigate the large Péclet number limit which is equivalent with a thin solid region.

In this limit $P e \gg 1$ the ratio $F_{R}^{\prime}\left(\lambda_{n}^{2} / P e\right) / F_{R}\left(\lambda_{n}^{2} / P e\right) \simeq \lambda_{n}^{2}$ $(1-R) R / P e$. Thus, one has merely to solve the simplified eigenproblem

$$
\begin{gathered}
-\Phi\left(\frac{1}{2}-\frac{\lambda_{n}}{4}, 1, \lambda_{n}\right)+2 \Phi^{\prime}\left(\frac{1}{2}-\frac{\lambda_{n}}{4}, 1, \lambda_{n}\right) \\
=\frac{k^{\mathrm{II}}}{k^{\mathrm{I}}} \frac{R(R-1)}{P e^{2}} \lambda_{n}^{3} \Phi\left(\frac{1}{2}-\frac{\lambda_{n}}{4}, 1, \lambda_{n}\right),
\end{gathered}
$$

to find the eigenvalue $\lambda_{n}$. Direct numerical results of the coupled problem (1) have been carried out by [2] in this limit and have shown that the typical entry length in Fig. 1b configuration depends on a single parameter $I$,

$I=\frac{k^{\mathrm{II}}}{k^{\mathrm{I}}} \frac{R(R-1)}{2 P e^{2}}$,

only. Eigenvalue problem (8) shows precisely the same dependence on the geometrical, physical and hydrodynamical parameters of the problem.

\subsection{Forward eigenvalue $\lambda_{+n}$}

Forward eigenvalues are associated with the downstream decrease of temperature in Fig. 1a configuration.
In the limit of infinite Péclet number, eigenvalues $\lambda_{+n}$ have finite values defined by the following transcendental equations:

$$
\Phi\left(\frac{1}{2}-\frac{\lambda_{+n}^{\infty}}{4}, 1, \lambda_{+n}^{\infty}\right)=2 \Phi^{\prime}\left(\frac{1}{2}-\frac{\lambda_{+n}^{\infty}}{4}, 1, \lambda_{+n}^{\infty}\right) .
$$

In this limit $\lambda_{+n}$ does not depend on the three parameters of the problem $R, P e$ and $k^{\mathrm{I}} / k^{\mathrm{II}}$ and exactly coincides with the Neumann-Graetz problem eigenvalues. For large but finite Péclet numbers and for a small aspect ratio, one can linearize (8) to get the asymptotic behavior of the eigenvalues, which only depends on I

$\lambda_{+n}-\lambda_{+n}^{\infty}=\frac{2 \lambda_{+n}^{\infty 3} \alpha_{n}}{\pi} \frac{k^{\mathrm{II}} R(1-R)}{k^{\mathrm{I}} P e^{2}}$,

where $\alpha_{n}=1 /\left(\left(\frac{1}{2}-\frac{\lambda_{+n}^{\infty}}{4}\right)\left(\frac{3}{2}-\frac{\lambda_{+n}^{\infty}}{4}\right) \Phi\left(\frac{5}{2}-\frac{\lambda_{+n}^{\infty}}{4}, 3, \lambda_{+n}^{\infty}\right)-(1 / 4) \Phi\right.$ $\left.\left(\frac{1}{2}-\frac{\lambda_{+n}^{\infty}}{4}, 1, \lambda_{+n}^{\infty}\right)\right)$. When the diffusivity ratio decreases to zero, or when the solid radius cylinder decreases to 1 , the transport in regions I and II becomes decoupled and, of course, one finds the asymptotic Graetz-Neumann solution. Again, the asymptotic validity of the linearization (11) is interesting to compare with numerical computations. The full eigenproblem (7) has been solved with a Newton method using the numerical evaluation of the confluent hyper-geometric function proposed in [4]. The eigenvalues have been tracked with a continuation method. Fig. $2 \mathrm{a}$ and $\mathrm{b}$ display the behavior of the first eigenvalue $\lambda_{+1}$. Fig. 2a shows that when the diameter of the outer cylinder is twice as large as that of the inner one and when diffusivity is the same in both, linearization (11) gives good approximation for the first eigenvalue even for moderate values of the Péclet number. A careful inspection of the numerical values represented in Fig. 2a shows that the prediction (11) is already $4 \%$ accurate for Péclet number value as low as 30 . Fig. 2 b compares the proposed scalings for the eigenvalues as a function of the cylinder aspect ratio $R$. It shows that even if the asymptotic prediction is supposed to hold for $1-R \ll 1$, it is still very good for large values of $R$ when the Péclet number is large.

\subsection{Backward eigenvalue $\lambda_{-n}$}

Backward eigenvalues are associated with the upstream decrease of temperature in Fig. 1b configuration. The eigenvalue $\lambda_{-1}$ has previously been studied with direct numerical simulations by [2] when the outer cylinder is thin $(R-1 \ll 1)$. They have described the temperature decrease observed in the domain $\zeta<0$ of Fig. 1b configuration. Using these numerical simulations as an heuristic base, Piva [7] found the simplified eigenproblem (8) by averaging the temperature field in the solid. In this domain, fluid convection in region I and solid diffusion in region II play antagonist roles for the temperature rises from the far field applied reference to the $\zeta=0$ heat source. The more convection there is, the smaller the temperature of establishment length will be, while conversely, the more diffusion 

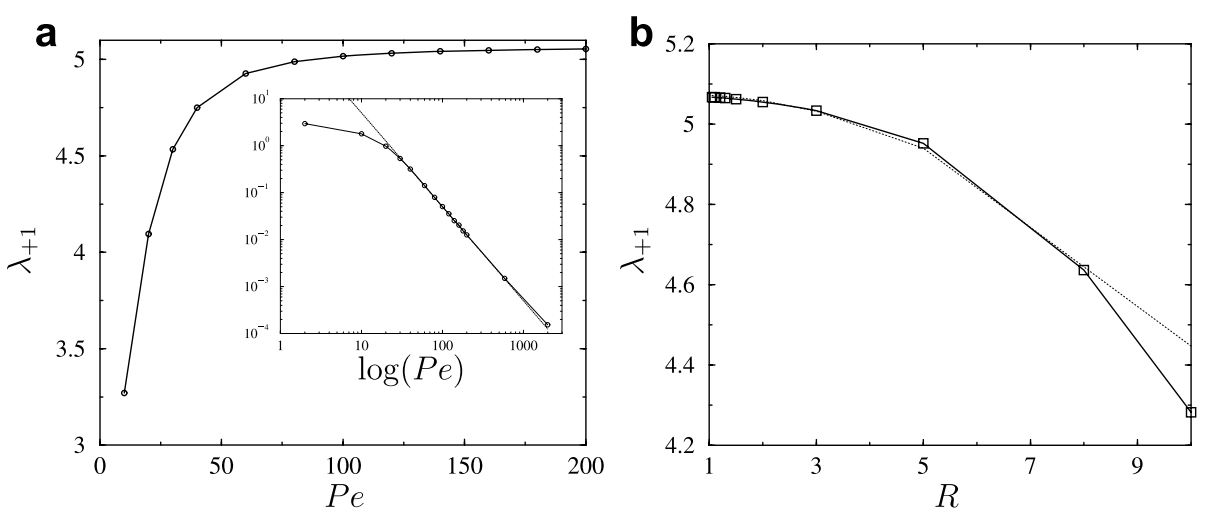

Fig. 2. (a) First eigenvalues $\lambda_{+1}$ versus $P e$, for $R=2, \frac{k^{1}}{k^{\mathrm{I}}}=1$. The inset represents a $\log -\log$ representation of the difference $\lambda_{+1}-\lambda_{+1}^{\infty}$ with $\lambda_{+1}^{\infty}=5.067505$ versus the Péclet number; the asymptotic (11) is represented with dotted lines. (b) Same conventions as in figure (a) versus the aspect ratio $R$ for $P e=200$. Dotted line illustrates the asymptotic (11).

in the solid, the larger the distance for the temperature to be established. Thus establishing length tends to zero as the Péclet number goes to infinity while conversely the eigenvalue $\lambda_{-1}$ tends to infinity. The divergence of this backward eigenvalue with the Péclet number is illustrated in Fig. 3a. The full eigenvalue problem (7) is solved here and represented in Fig. 3a. In Section 5 it is compared to the numerical solution of the approximated problem (8) and to experimental measurements. On the other hand, the approximated eigenproblem (8) can be used to find an asymptotic expression for the eigenvalue $\lambda_{-n}$ with the Péclet number

$\frac{\Phi^{\prime}\left(1 / 2-\lambda_{-n} / 4,1, \lambda_{-n}\right)}{\Phi\left(1 / 2-\lambda_{-n} / 4,1, \lambda_{-n}\right)}=\frac{1}{2}+\frac{k^{\mathrm{II}} R(R-1)}{k^{\mathrm{I}} 2}\left(\frac{\lambda_{-n}^{3}}{P e^{2}}\right)$.

Nevertheless it is not easy to get the asymptotic behavior of the confluent hyper-geometric function when both its first parameter and its variable tend to infinity. Erdélyi and Swanson [3] have thoroughly discussed the numerous asymptotic behavior of function $\Phi(a, b, \lambda)$ in this limit, for fixed values of the ratio $a / 4 \lambda$. In the presently interesting limit where $4 a / \lambda \rightarrow 1$, their results show that the logarithmic derivative of the confluent geometric function does not diverge algebraically when $a \rightarrow \infty$ and $\lambda \rightarrow \infty$. The numerical calculation confirms this result leading to logarithmic dependence on $\lambda_{-1}$. As illustrated in Fig. $3 \mathrm{~b}$ the asymptotic behavior of the first upstream eigenvalue $\lambda_{-1}$ display a logarithmic dependence with the single parameter $I$, which collapse into a single master curve when changing the aspect ratio parameter $R$. This leads to the following expression of the backward eigenvalue dependence on the Péclet number:

$\lambda_{-1}=I^{-1 / 3}(0.026 \ln I+0.915)$.

The linear behavior with the logarithm of parameter $I$ requires two constants that have been obtained from fitting the numerical computation sketched in Fig. $3 b$.

\section{Fully developed transfer}

The Nusselt number is defined by the non-dimensional flux between region I and II:

$N u(\zeta)=\frac{1}{\Theta_{b}(\zeta)-\Theta_{\mathrm{w}}(\zeta)} \frac{\partial \Theta}{\partial n}$

where $\Theta_{\mathrm{w}}=\Theta(1, \zeta)$ is the wall temperature and $\Theta_{b}$ is given by

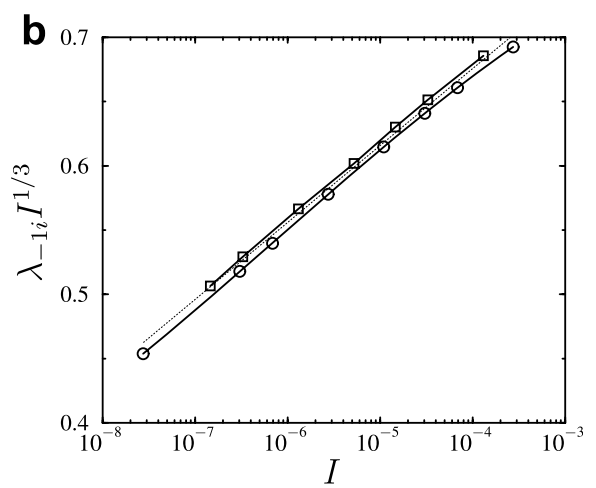

Fig. 3. (a) Imaginary part of the first backward eigenvalue $\lambda_{-1}$ versus $P e$, for $R=1.1$ with circle symbols and $R=1.05$ for square symbols with $\frac{k^{I}}{k^{11}}=1$. (b) Same conventions for $\lambda_{-1 i} I^{1 / 3}$ versus parameter $I$ in semi-logarithmic representation. 
$\Theta_{b}=\frac{1}{S^{\mathrm{I}} U} \int_{S^{\mathrm{I}}} u \Theta^{\mathrm{I}} \mathrm{d} S^{\mathrm{I}}, \quad U=\frac{1}{S^{\mathrm{I}}} \int_{S^{\mathrm{I}}} u \mathrm{~d} S^{\mathrm{I}}$,

where $S^{\mathrm{I}}$ is the region I disk surface, and $\Theta_{b}$ is the flow weighted averaged temperature or "mixing cup" temperature. Another definition is sometimes found in the literature omitting the $\Theta_{\mathrm{w}}(\zeta)$ term of the denominator. We have kept this definition here to be able to compare with some experimental data in the backward configuration. Looking for a simplified far field expression for the Nusselt number one finds that its fully developed asymptotic behavior reaches a constant value which depends only on the first eigenfunction and eigenvalue. This can be done from averaging with a "mixing cup" weight the convection-diffusion equation (1) in the liquid. This leads to a relation between $\Theta_{b}$ and the transverse temperature variation at the inner wall:

$\frac{1}{4} P e \partial_{\zeta} \Theta_{b}=\partial_{\eta} \Theta(1, \zeta)$.

Using this relation and solution (6), one can then find the fully developed approximation of these fields in the limit where $|\zeta| \gg 1$,

$$
\begin{aligned}
\Theta_{\mathrm{w}} & \simeq \theta_{1} \mathrm{e}^{-\lambda_{1} / 2} \mathrm{e}^{-\lambda_{1}^{2} \zeta / P e} \Phi\left(\frac{1}{2}-\frac{\lambda_{1}}{4}, 1, \lambda_{1}\right), \\
\Theta_{b} & \simeq-4 \frac{\theta_{1}}{\lambda_{1}} \mathrm{e}^{-\lambda_{1} / 2} \mathrm{e}^{-\lambda_{1}^{2} \zeta / P e}\left(2 \Phi^{\prime}\left(\frac{1}{2}-\frac{\lambda_{1}}{4}, 1, \lambda_{1}\right)\right. \\
& \left.-\Phi\left(\frac{1}{2}-\frac{\lambda_{1}}{4}, 1, \lambda_{1}\right)\right) .
\end{aligned}
$$

From these expressions, one is then able to find an explicit formulation of the fully developed Nusselt number from using definition (14)

$N u=\frac{\lambda_{1}}{\frac{\Phi\left(\frac{1}{2}-\frac{\lambda_{1}}{4}, 1, \lambda_{1}\right)}{-2 \Phi\left(\frac{1}{2}-\frac{\lambda_{1}}{4}, 1, \lambda_{1}\right)+4 \Phi^{\prime}\left(\frac{1}{2}-\frac{\lambda_{1}}{4}, 1, \lambda_{1}\right)}+\frac{2}{\lambda_{1}}}$.

Although explicit, this expression can be even further simplified in the context of the large Péclet numbers and small solid diameter previously considered in Eq. (8) for which it simply reads

$N u \simeq 4 \lambda_{1}^{4} I$,

where, again, the limit $I=\frac{k^{I I}}{k^{I}} \frac{R(R-1)}{2 P e^{2}} \ll 1$ is considered.

Now, using the asymptotic expression obtained in (11) leads to the explicit formula, in terms of the physical, geometrical, and hydrodynamical parameters of the problem, for the forward eigenvalue

$N u_{+}=2 \lambda_{+1}^{\infty} 4\left(1-8 \frac{\lambda_{+1}^{\infty} \alpha_{1}}{\pi} \frac{k^{\mathrm{II}}}{k^{\mathrm{I}}} \frac{R(R-1)}{P e^{2}}\right) \frac{k^{\mathrm{II}}}{k^{\mathrm{I}}} \frac{R(R-1)}{P e^{2}}$.

It is interesting to note that, to the leading order neglecting $\mathrm{O}\left(1 / P e^{2}\right)$ effects, the forward transfer is not increasing with the Péclet number. On the contrary it decreases quadratically with this parameter. This result is not intuitive since increasing the flow enhances the temperature longitudinal gradient. The typical axial decreasing length of the temperature field $\ell_{+}$scales as $\ell_{+} \sim P e / \lambda_{+1}^{2}$. When the fluid is increased this length asymptotically increases linearly with the Péclet number. A side effect of this increase is nevertheless that, conversely, normal gradients shrink when the stationary temperature field is stretched by convection, so that the transverse gradient scales as $1 / \ell_{+}$.

Most of the temperature variations are concentrated at the tube center, and decrease rapidly to reach a quasi-constant value near the tube inner wall. In this limit, the temperature difference between the liquid and the wall is thus grossly proportional to $\ell_{+}$. Moreover, since the radial temperature gradient scales as $1 / \ell_{+}$, the Nusselt number which is the product between the inverse of temperature difference and the temperature gradient should then scale as $N u_{+} \sim 1 / \ell_{+}^{2}$, so that $N u_{+} \sim 1 / P e^{2}$. These physical arguments thus allows to recover the leading order scaling (20). Both asymptotic are compared with the numerical solution of (19) in Fig. 4. One can observed the convergence to the quadratic leading order dependence with the Péclet number found in (20) in Fig. 4a for different values of the aspect ratio $R$. This figure again shows that the range of value of $R$ for which the asymptotic regime applies can be quite broad reaching values as large as 5 , for moderate value of the Péclet number of the order of 100 . Fig. $4 \mathrm{~b}$ compares the leading and first order asymptotic behaviors given above, while all different sets collapse into a single master curve when plotted as a function of parameter $I$, in the limit of small values of this parameter.

In the case of the backward Nusselt number, an asymptotic expression can also be obtained from (13) and (19), in the limit of small values of parameter $I$, so that one finds

$N u_{-}=I^{-1 / 3} 4(0.026 \ln I+0.915)^{4}$.

This asymptotic behavior leads to larger and larger Nusselt numbers when increasing the Péclet number, as opposed to the forward case. This trend is confirmed from the computation of the Nusselt number from (19) depicted in Fig. 5a, from which it can be observed that the Nusselt number reaches values an order of magnitude larger than in the forward case, even for moderate values of the Péclet number. The transfer from the solid to the fluid is thus much more important in the backward direction, even if the lengthscale $\ell_{-}$associated with the typical axial decreasing length of the temperature field is much more smaller. From the definition of parameter $I$ given in (9), it is easy to see that the leading order scaling in Péclet, is $N u_{-} \sim P e^{2 / 3}$ up to logarithmic corrections. This leading order behavior can be grossly understood from the same physical argument previously developed for the forward Nusselt number, indicating that in the backward case it should scale as $N u_{-} \sim 1 / \ell_{-}^{2}$. Since $\ell_{-} \sim P e / \lambda_{-1}^{2} \sim P e^{-1 / 3}$, we then find that the transfer should scale as $N u_{-} \sim P e^{2 / 3}$. The logarithmic correction that have been previously obtained should be viewed as a particularity of the cylindrical geometry that 

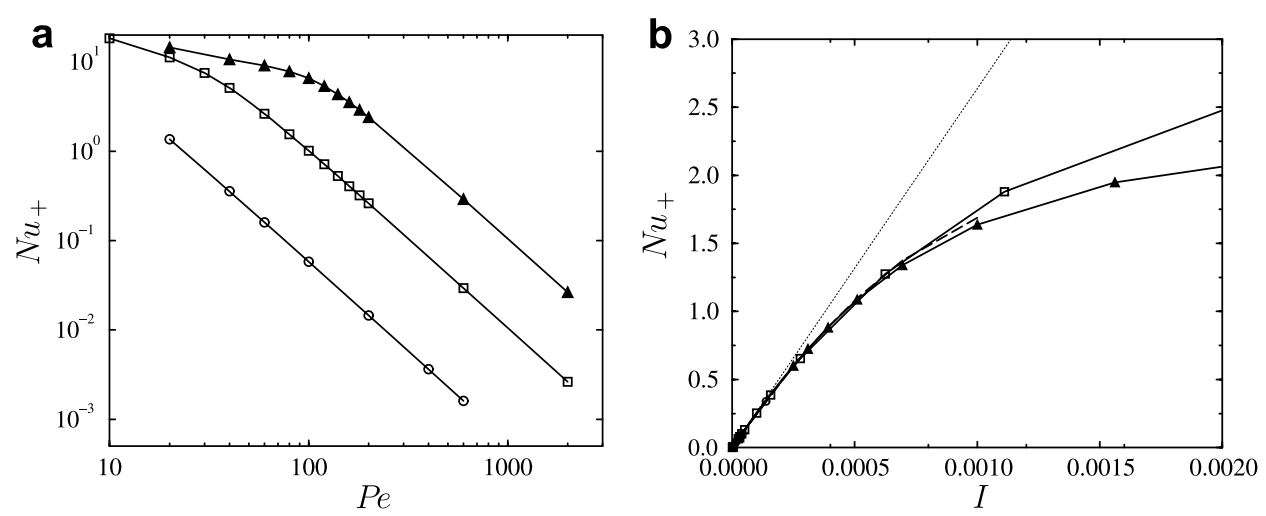

Fig. 4. Fully developed forward Nusselt number for different parameters. Bullet symbols are associated with $R=1.1$, square symbols for $R=2$ and black triangular symbols with $R=5$. (a) Versus the Péclet number. (b) Versus the parameter $I$ defined in (9). The leading order asymptotic behavior (20) is shown with dotted lines, while the next order is represented with long dashed lines.

has been chosen here, and should not appear in the similar planar problem. It is also interesting to note that the validity range of the asymptotic behavior (21) is much more restrain than in the forward case as illustrated in Fig. 5 b. For moderate values of the Péclet number such as 100, the value of $R$ for which it applies are not larger than 1.1. One can observed for instance that the behavior of the Nusselt number associated with $R=2$ on Fig. 5 b does not collapse on the same master curve associated with the asymptotic limit. In the limit of extremely small values of parameter $I$ it should nevertheless merge with the other curves.

\section{Comparison with experiments}

It is interesting to compare theory and experiments using the single parameter $I$ to collapse different geometrical, physical or hydrodynamical situations when the solid cylinder wall is small. To our knowledge, only experimental measurements in Fig. 1b configuration are available in the literature. Using a copper tube the radius of which is onetenth of the inner cylinder, and using water as a working fluid [8] carried out a large number of experiments for
Péclet numbers ranging from 1180 to 5000 . The heat conductivity of copper being much larger than that of water, the conductivity ratio was in their case equal to $k^{\mathrm{II}} /$ $k^{\mathrm{I}}=549 \pm 19$. Two major parameters are interesting to compare with the experimental measurements. First, let us consider the longitudinal entry length $\ell_{-}$(called $m$ in [8]) characterizing the exponential decrease in temperature. This parameter is determined from the log-linear best slope fit of the temperature variations measured with thermocouples placed along the longitudinal direction of the external face of the solid cylinder. The 25 data measurements of [8] are represented in Fig. 6a and compared with theoretical predictions. A prediction in the case of the thin solid wall limit is derived from solving Eq. (8). It is interesting to note that this prediction is in very good agreement with experimental measures, as already found in [7]. This approximated theory compares very closely to the exact solution of Eq. (7) in the case of radius ratio $R=10^{-2}$. A difference between this approximated theory and the exact one is nevertheless observed in the case of $R=10^{-1}$ which corresponds to experimental conditions. Thus, even if the theoretical predictions are very satisfyingly close to experiments, a discrepancy of about $10 \%$ is observed, which
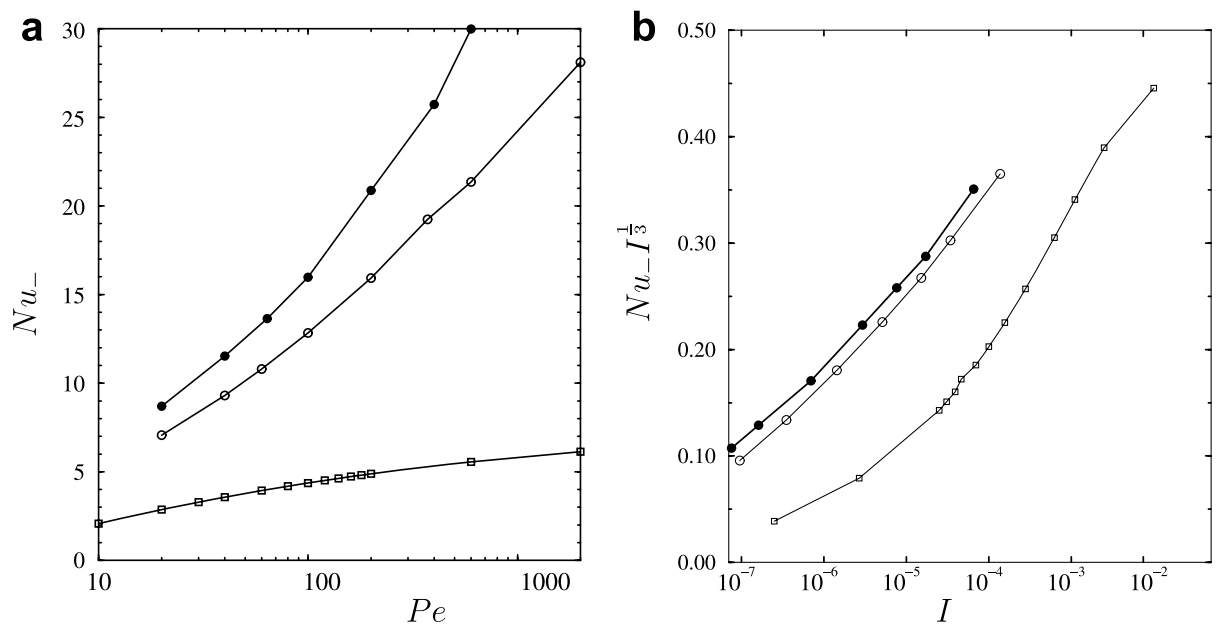

Fig. 5. Fully developed backward Nusselt number for different parameters. Black bullet symbols are associated with $R=1.05$, while white ones are for $R=1.1$ and square symbols for $R=2$. (a) Versus the Péclet number. (b) Versus the parameter $I$ defined in (9). 

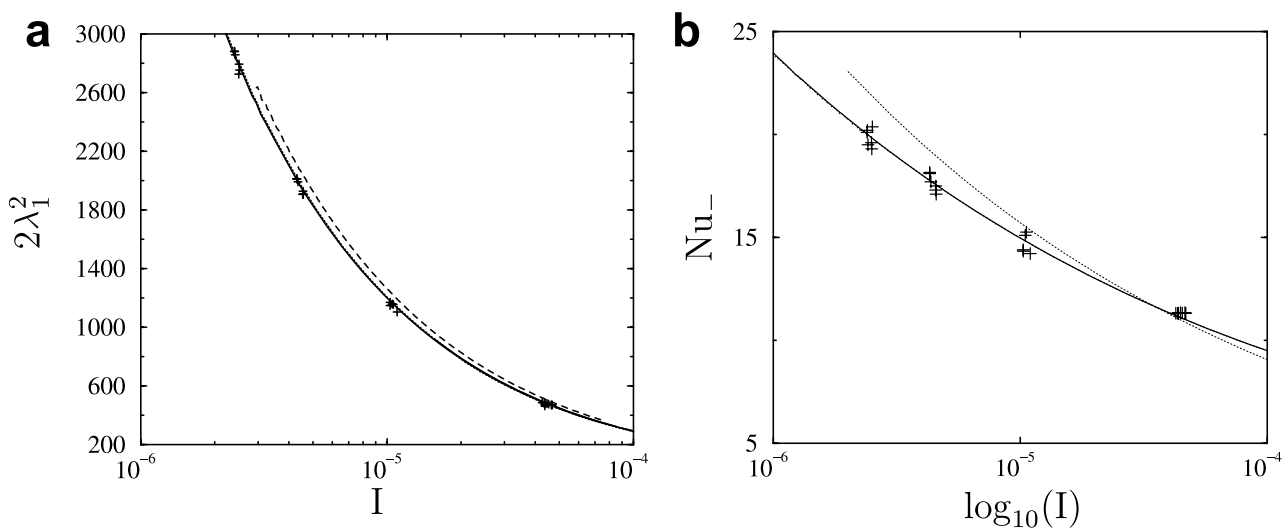

Fig. 6. (a) $2 \lambda_{1}^{2} \equiv m$, parameter used in [8] plotted versus the non-dimensional parameter $I$. The numerical solution of (7) is represented with continuous line for $1-R=10^{-2}$ and dashed lines for $1-R=10^{-1}$. Numerical solution of the approximated eigenvalue problem (8) represented with dotted lines. The experimental results of [8] are represented with crosses. (b) Same conventions as figure (a) for the comparison between theory and experiments for the Nusselt number.

could be within the experimental error bars. Another interesting parameter to compare with the experiments is the Nusselt number represented in Fig. 6b. The Nusselt number cannot be directly measured in the experiments; it is rather extrapolated from temperature measurements. Here again, theory and experiments can compare in a satisfactory way, within a $10 \%$ difference.

\section{Conclusion}

We found simple asymptotic expression for the first upward and backward eigenvalues of the extended Graetz problem. Those results should be useful for testing numerical estimation or experimental results in the limit of large Péclet number.

\section{Acknowledgments}

We thanks M. Quintard and A. Bergeon for stimulating discussions.

\section{References}

[1] M. Abramowitz, I. Stegun, Handbook of Mathematical Functions, Dover Publication, Inc., New-York, 1972.
[2] M.A. Cotton, J.D. Jackson, in: R.W. Lewis, K. Morgan (Eds.), Numerical Methods in Thermal Problems, vol. IV, Pineridge Press, Swansea, 1985, pp. 504-515.

[3] A. Erdélyi, C.A. Swanson, Asymptotics forms of Wittakers confluent hypergeometric functions, Mem. Am. Math. Soc. 25 (1957) 929.

[4] M. Nardin, W.F. Perger, A. Bhalla, Algorithm 707 conhyp: a numerical evaluator of the confluent hypergeometric function for complex arguments of large magnitudes, ACM Trans. Math. Software 18 (3) (1992) 345-349.

[5] E. Papoutsakis, D. Ramkrishna, H.C. Lim, The extended Graetz problem with Diriclet wall boundary conditions, Appl. Sci. Res. 36 (1980) 13-34.

[6] E. Papoutsakis, D. Ramkrishna, H.C. Lim, The extended Graetz problem with prescribed wall flux, AIChE J. 26 (1980) 779-787.

[7] S. Piva, Axial wall conduction preheating effects in high Péclet number laminar forced convection, Int. J. Heat Mass Transfer 39 (16) (1996) 3511-3517.

[8] S. Piva, G. Pagliarini, Estimation of unknown parameters from exponential heating of laminar flow, Exp. Therm. Fluid Sci. 8 (1994) $21-27$.

[9] A. Silva Telles, E.M. Queiroz, G. Elmôr Filho, Solutions of the extended Graetz problem, Int. J. Heat Mass Transfer 44 (2) (2001) $471-483$.

[10] B. Weigand, M. Kanzamar, H. Beer, The extended Graetz problem with piecewise constant wall heat flux for pipe and channel flows, Int. J. Heat Mass Transfer 44 (20) (2001) 3941-3952.

[11] B. Weigand, D. Lauffer, The extended Graetz problem with piecewise constant wall temperature for pipe and channel flows, Int. J. Heat Mass Transfer 47 (24) (2004) 5303-5312. 